\title{
Scale Summarized and Focused Browsing of Primitive Visual Content
}

\author{
Xenophon Zabulis ${ }^{12}$, Jon Sporring ${ }^{3}$, and Stelios C. Orphanoudakis ${ }^{12}$ \\ 1 Foundation for Research and Technology - Hellas, \\ Vassilika Vouton, P.O. Box 1385, \\ GR-71110 Heraklion, Crete, Greece \\ \{zabulis, orphanou\}@ics.forth.gr \\ 2 Department of Computer Science, \\ University of Crete, \\ P.O. Box 1470, \\ GR-71409 Heraklion, Crete, Greece \\ 3 3D-Lab, School of Dentistry, \\ University of Copenhagen, \\ Nørre Allé 20, \\ DK-2000 Copenhagen, Denmark \\ sporring@asters. lab3d.odont.ku.dk
}

\begin{abstract}
A study of local scale in images demonstrates that image features reside in different scales. Based on this observation a framework for the classification of features with respect to scale is proposed, linearly combining the visual impression of features at different scales. The proposed framework and a derived methodology are applied to typical feature extraction tasks, and in the generic case of estimating multiple scale feature distributions, as a tool for the identification of images of similar visual content. A possible formulation of queries for retrieving images by primitive visual content, taking scale into account, is also discussed.
\end{abstract}

\section{Introduction}

Structure in digital images resides somewhere between two scales, one defined by the sampling interval, and the other by image size. Thus, in order to focus attention at structures of different sizes, it is important to have the ability to select the appropriate scale. In general, scale selection finds application in almost all computer vision tasks such as image processing, feature detection, and image description. Therefore, it is central issue in the context of image retrieval by content.

\footnotetext{
${ }^{1}$ This research was carried out during Jon Sporring's term as a VIRGO postdoctoral fellow at ICS-FORTH and was funded in part by EC Contract No. ERBFMRX-CT96-0049 under the TMR Programme of the CEC. (http://www.ics.forth.gr/virgo)
} 
It is possible and certainly useful to detect a single scale that matches the size of a structure. Nevertheless, several significant scales may be present at a single image point. Furthermore, the existing algorithms for singular scale selection are rather complicated and employ feature tracking at different scales $[1,6]$, which may be intractable or at least unstable without a substantial number of scales used.

The application of feature extraction in the identification of images with similar primitive visual content is straightforward (but not simple), if context is neglected. The goal is to identify images that contain similar visual features or feature combinations, probably also taking into account their spatial arrangement. If features are classified with respect to their scale then more refined visual queries may be formulated. For example, a query could primarily aim at the retrieval of images of more abstract or dominant scene similarity, implying that feature comparison and matching should take place at coarse scales. Classifying features with respect to their scale should not only reduce similar feature retrieval time, but also support the formulation of more qualitative queries. In the same line of thought, feature scale may be used as a classification attribute in the perceptual grouping of image features into meaningful visual entities.

In the present work, Scale Summary Representation (SSR) and Scale Focusing (SF) are introduced, the first as a tool for representing feature information from all scales and the second as a classifier of features with respect to scale. The basic idea in SSR is the representation of information from all scales, into a single image, based on feature appearance intensity at each scale. SF smoothly restricts this process into a neighborhood of scales in order to extract features that reside only in these scales. Typically attention will be shifted to the dominant modes of scale observation, where the feature is best detected.

This paper is organized as follows: In Section 2, the theoretical definition of SSR and SF is defined along with details of how these tools may be applied to feature extraction. Section 3 presents experimental results from the domain of image feature extraction. In Section 4, the application of SSR to the estimation of feature distributions and image retrieval by content is discussed. Finally, conclusions and plans for future work are discussed in Section 5.

\section{A Summary of Scale}

Scale Summary Representation (SSR) is introduced as a method to summarize image features over scale for different classes of images. Such classes include the linear scale space (Gaussian smoothed images, see [6,12] for an overview), the families of angular content at various scales, etc.. The scale summarized image is defined as a weighted sum,

$$
\begin{gathered}
J(\boldsymbol{x})=\sum_{\tau} w(\boldsymbol{x}, \tau) A[L(\boldsymbol{x}, \tau)], \\
\sum_{\tau} w(\boldsymbol{x}, \tau)=1,
\end{gathered}
$$


where $\tau=\log t$ is the logarithmic scale parameter, $I$ the original image, $A$ is a feature operator, $L$ the image scale space (given by $L(\boldsymbol{x}, \tau)=G(\boldsymbol{x}, t) * I(\boldsymbol{x})$, with $\left.G(\boldsymbol{x}, t)=\exp \left(-|\boldsymbol{x}|_{2}^{2} /(4 t)\right) /(\sqrt{4 \pi t})\right)$, and $w$ the probability of feature presence. The weight function $w$ is henceforth called the scale selector. A simple scale selector for image edge related features (such as edges, orientation or corners) is the scale normalized square gradient $\operatorname{Grad}^{2}(\boldsymbol{x}, \tau)=t\left(L_{x}^{2}(\boldsymbol{x}, \tau)+L_{y}^{2}(\boldsymbol{x}, \tau)\right)[6]$. Normalizing the sum yields the following scale selector:

$$
w_{\text {edge }}(\boldsymbol{x}, \tau)=\frac{1}{k_{\text {edge }}(\boldsymbol{x})} h\left(t \operatorname{Grad}^{2}(\boldsymbol{x}, \tau)\right) .
$$

where the function $k_{\text {edge }}(\boldsymbol{x})=\int_{0}^{\infty} h\left(t \operatorname{Grad}^{2}(\boldsymbol{x}, \tau)\right) d \tau$ is the normalizing function at each spatial point, and $h$ is any strictly increasing function, chosen according to the nature of the feature detector. For simplicity, $h(x)=x$ is used throughout this paper. An example of a scale selector for intensity blob related features (such as gray level surfaces) is the scale-normalized Laplacian:

$$
w_{\mathrm{blob}}(\boldsymbol{x}, \tau)=\frac{1}{k_{\mathrm{blob}}(\boldsymbol{x})} h\left(t\left|L_{x x}(\boldsymbol{x}, \tau)+L_{y y}(\boldsymbol{x}, \tau)\right|\right),
$$

where the normalization function is $k_{\mathrm{blob}}(\boldsymbol{x})=\int_{0}^{\infty} h\left(t\left|L_{x x}(\boldsymbol{x}, \tau)+L_{y y}(\boldsymbol{x}, \tau)\right|\right) d \tau$. Fig. 1 illustrates the SSR response of these two scale selectors, using them as the feature operator $A$ in Eq. 1.
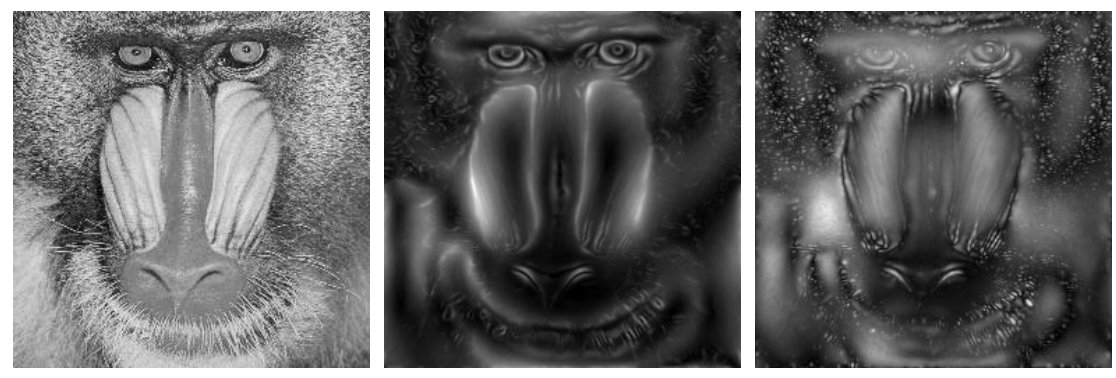

Fig. 1. An image (left) and the SSR scale selector response for edges (middle) and blobs (right).

For summarizing feature content restricted to a neighborhood of scales, Scale Focusing (SF) is introduced as the multiplication of the scale selector function with the Gaussian function at each pixel, as

$$
w_{m, s}^{\prime}(\boldsymbol{x}, \tau)=\frac{1}{\sqrt{4 \pi s(\boldsymbol{x})}} \exp \left(-\frac{(\tau-m(\boldsymbol{x}))^{2}}{4 s(\boldsymbol{x})}\right)
$$


where $m$ is the scale of interest and $s$ the width of the scale neighborhood. A typical application of SF is the detection of the dominant scale at each pixel. In this case the Gaussian is centered at the maximum of the scale selector function.

Fig. 2 (top row) shows a synthetic image and the blob scale selector for the central dot (with the horizontal axis indicating scale). Three modes are observed corresponding to the three significant scales. The first three images of Fig. 2 (bottom row) illustrate the results of SF on these. The last image represents information from all scales in one image, using Eqs. (1) and (4). As observed, the response for fine scale dominates over the implicitly formed blobs at coarser scales. The importance of different scales may be defined by adjusting function $h$ to reflect the bias.
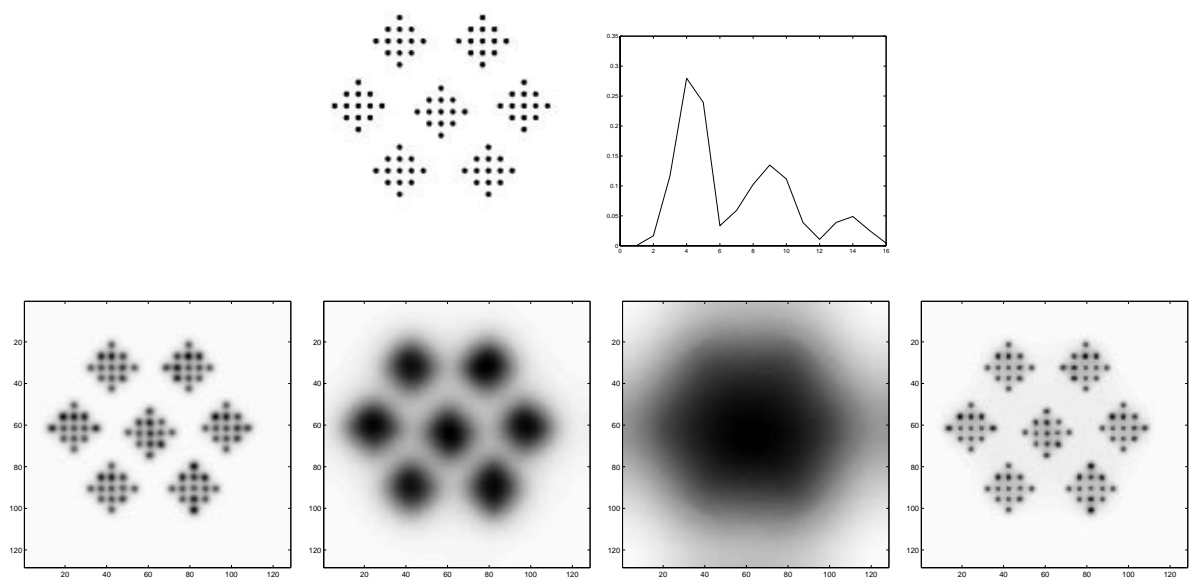

Fig. 2. An image and the blob scale selector for the image point corresponding to the central dot (top row). Three cases of SF and SSR of local scale for blob detection (bottom row, from left to right).

\section{Feature Detection Applications}

Summarizing feature detection information, carried out at all image scales, is expected to enhance the performance of methods operating at a fixed scale. The explicit selection of one scale for each image pixel is subject to computational instability because of noise and scale discretization, and typically yields unsmooth results.

The framework of SSR is applied to two categories of feature detection tasks: a) features derived from the image gradient, such as the detection of edges, linear feature orientation, and corners, and b) intensity blobs. In the latter category, SSR is applied to image smoothing as a representative case of blob detection. 


\subsection{Image Gradient Derived Features}

By scale summarizing image gradients, a scaleless edge detection result is obtained, detecting edges at all scales. Using Eq. 3 as the feature operator $A$ in Eq. 1 results in an image that scale summarizes edge information, as shown in Fig. 1 (middle image). To illustrate the effect of SF, as well as the discrimination among edges at different scales, Fig. 3 shows the results of SF on a fine and a coarse scale, where vertical edges are robustly detected at the higher scale. The last image shows the result of SSR.
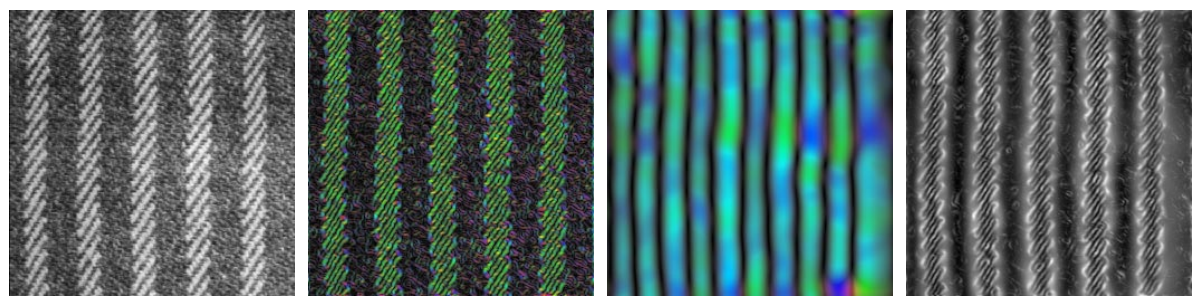

Fig. 3. An image and the SF of its gradient on a fine and coarse scale. The last image shows the scale summarized gradients (from left to right). In the two middle images, when viewed in color, local orientation is linearly mapped to the color hues.

An important primitive visual feature is the local orientation of edges and linear features. A robust measure of local orientation is the direction of the eigenvector corresponding to the largest eigenvalue of the structure tensor [4]:

$$
\mu\left(\boldsymbol{x}, \tau_{1}, \tau_{2}\right)=\left[\begin{array}{cc}
G\left(\boldsymbol{x}, \tau_{2}\right) * L_{x}\left(\boldsymbol{x}, \tau_{1}\right)^{2} & G\left(\boldsymbol{x}, \tau_{2}\right) * L_{x}\left(\boldsymbol{x}, \tau_{1}\right) L_{y}\left(\boldsymbol{x}, \tau_{1}\right) \\
G\left(\boldsymbol{x}, \tau_{2}\right) * L_{x}\left(\boldsymbol{x}, \tau_{1}\right) L_{y}\left(\boldsymbol{x}, \tau_{1}\right) & G\left(\boldsymbol{x}, \tau_{2}\right) * L_{y}\left(\boldsymbol{x}, \tau_{1}\right)^{2}
\end{array}\right]
$$

The eigenvector corresponding to the largest eigenvalue will be robustly aligned along the gradient direction. As illustrated in Fig. 3, at fine scale a diagonal orientation dominates, while vertical dominates at a coarser scale. As scale increases, visual content with respect to orientation changes. Therefore, a full description of image content cannot be obtained by considering a single scale. In the two middle images local orientation is linearly mapped to the color hues. When viewed in grayscale, the scale focused gradient, upon which local orientation estimation is based, is shown.

Several measures exist for detecting corners in images. An overview of many of them may be found in [8]. Here the determinant divided by the trace of the structure tensor in Eq. (6) is used as the corner feature detector:

$$
C\left(\boldsymbol{x}, \tau_{1}, \tau_{2}\right)=\frac{l_{1}\left(\boldsymbol{x}, \tau_{1}, \tau_{2}\right) l_{2}\left(\boldsymbol{x}, \tau_{1}, \tau_{2}\right)}{l_{1}\left(\boldsymbol{x}, \tau_{1}, \tau_{2}\right)+l_{2}\left(\boldsymbol{x}, \tau_{1}, \tau_{2}\right)}
$$

where $l_{1}, l_{2}$ are the eigenvalues of $\mu\left(\boldsymbol{x}, \tau_{1}, \tau_{2}\right)$ and are functions are functions of $\boldsymbol{x}, \tau_{1}$, and $\tau_{2}$. A corner is an image feature, which depends on the existence of 
intensity or color edges and on the scale of investigation. For the computation, $\tau_{1}$ is fixed at a fine scale and $\tau_{2}$ is varied. Fig. 4 illustrates the detection of corners at a fine and a coarse scale, along with the SSR of corner information.
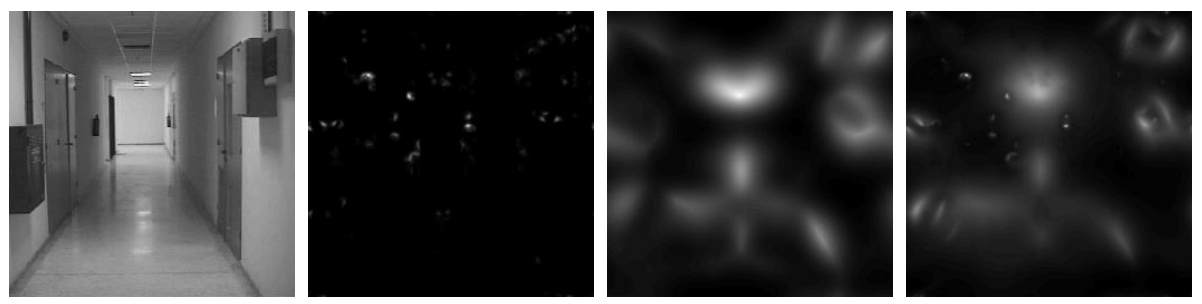

Fig. 4. An image and the detection of its corners at fine and coarse scale. The last image illustrates the scale summarized corner information (from left to right).

\subsection{Blobs / Local Neighborhood Estimation}

When summarizing blob information over scale, a scaleless blob detection result is obtained, detecting intensity regions at all scales. Using Eq. 4 as the feature operator $A$ in Eq. 1 results in an image that summarizes blob information over all scales, as shown in Fig. 1 (right image). Estimation of features dependent on some intensity blob, is related to the size and content of that region. SSR, based on the blob detector, favors scales that adapt to image structure. This way the contribution of scales, where samples are taken from the neighborhood of interest, dominates over others. In this category of applications blob size is estimated by Eq. (4).
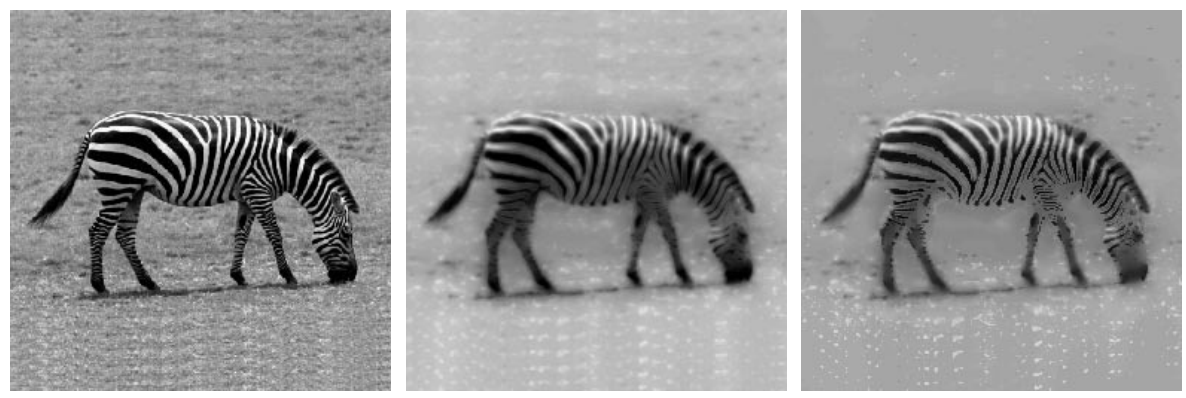

Fig. 5. Original image (left), SSR Smoothing (middle), Dominant SF Smoothing (right). 
Fig. 5 demonstrates the application of SSR to image smoothing. The middle image illustrates the smoothing results obtained with SSR, using the local structure estimator given by Eq. (4). The image structure is retained, since SSR favors the contribution of those scales corresponding to local image structure. The right image illustrates SF at the dominant mode of the scale selector, applied at pixel. It is demontrated that the dominating blobs' spatial features are preserved while the variance of their luminosity content decreases.

Iterative application of SSR smoothing yields a scale space for which it can be proven that the minimum and maximum values are monotonic increasing and decreasing functions of $n$ respectively. Qualitatively, the scale selector can be used to pick the next scale in linear scale-space. The family of images obtained with the iterative SSR or SF smoothing will decrease the variance of luminosity inside blobs while preserving their structure. Fig. 6 illustrates the evolution of an image over scale.
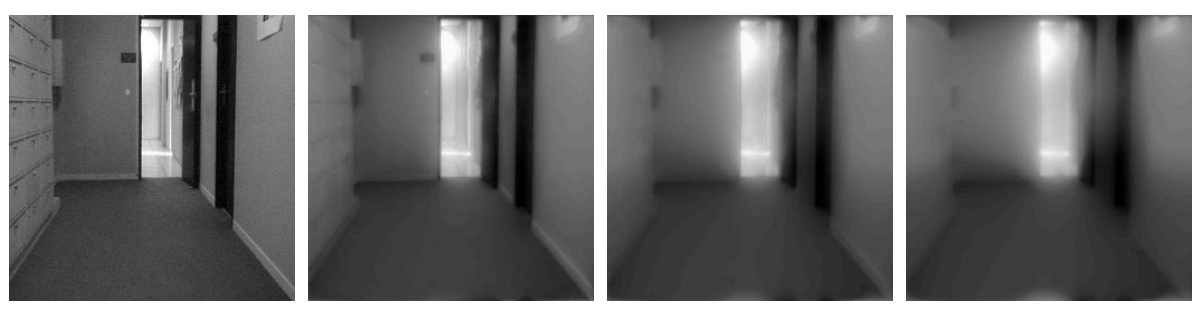

Fig. 6. Image evolution in scale space created by SSR smoothing (from left to right).

\section{Studying Feature Distributions for Image Retrieval by Content}

This section discusses the application of SSR and SF techniques in the generic study of spatial distributions of features and image retrieval by content. Through the multiscale estimation of feature distributions, a description of primitive visual content can be extracted in a statistically robust and stable way. SSR may be used for the dimemsionality reduction of the mutliscale feature distribution space, as well as for the normalization of similar distributions residing at different scales.

Estimation of local feature distributions is represented in a multidimensional scale space, where a local histogram is associated with each pixel neighborhood, for each image. Given the size $s$ of some sampling neighborhood in an image and computing the local histogram $h_{s}(\boldsymbol{x})$ of some feature for each image element, the multidimensional image of local histograms can be obtained. For simplicity of representation, elements of each image are mapped into vectors of dimensionality equal to the number of bins in the histogram. By varying the size of the 
sampling area a scale space of such images images is defined as $H(\boldsymbol{x}, \tau)$ [5], with $\tau$ indicating the scale index.

Summarizing the scale space into a single image of lower dimensionality is achieved with the use of Eq. (1), in the form of $J(x, \tau)=\sum_{\tau} w(x, \tau) H(x, \tau)$, while scale discrimination is achieved by focusing at the modes of the scale selector function which is chosen according to the feature sampled. The scale summarized data structure captures information from all scales with respect to the importance of each scale as defined by the scale selector. Similar feature distributions may be detected within the same image by clustering of distributions (using a suitable histogram distance function [3]).
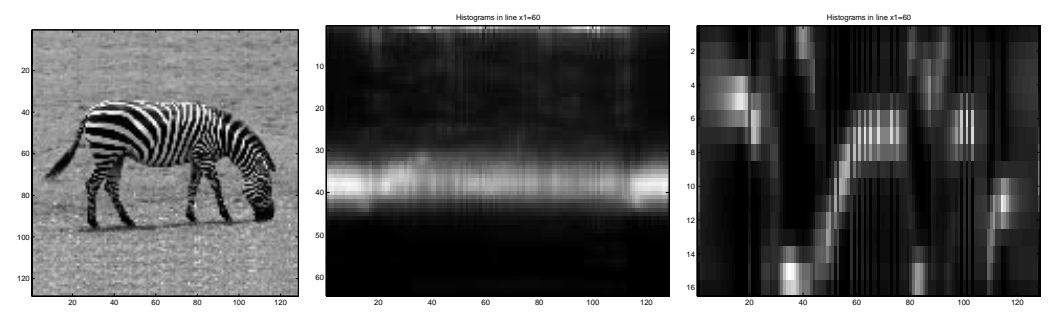

Fig. 7. An image and the visualization of the scale summarized feature distribution along the crentral line for luminosity (middle) and orientation (right).

The characterization of multiple feature distributions is a powerful que for identifying similar image regions in images. Fig. 7 (middle), shows the scale summarized histograms, vertically tiled, along the central image row. Notice that, due to the contribution of coarser scales and scale normalization, histograms from image column 30 to 110 are more similar, despite the local black / white variation (zebra stripes). Fig. 7 (right) illustrates the same information for the orientation feature. Distributions of small variance indicate a dominant direction, or parallelism. In the middle image of Fig. 8, intensity is inversely mapped with the variance of local orientation distribution, causing highly organized orientation distributions to stand out, as opposed to the right image, where luminosity is linearly mapped to the summarized gradient. Having in mind that visual content description is to serve the task of image retrieval by content, perceptual grouping rules [10] could also be incorporated into the clustering algorithm, so that clusters found would denote meaningful visual entities.

In an image retrieval by content application the system should search for clusters of similar distributions using multiple features. Judging the similarity of feature sets, in a perceptually correct and intuitive way, is critical for the appreciation of the retrieval result and an open issue [9]. Similarity of clusters may be judged afterwards by other criteria as well, such as spatial arrangement, size [11], etc.. Database queries may be formulated so that they would initially restrict search for similar distributions in coarse scales, where the image is abstract 

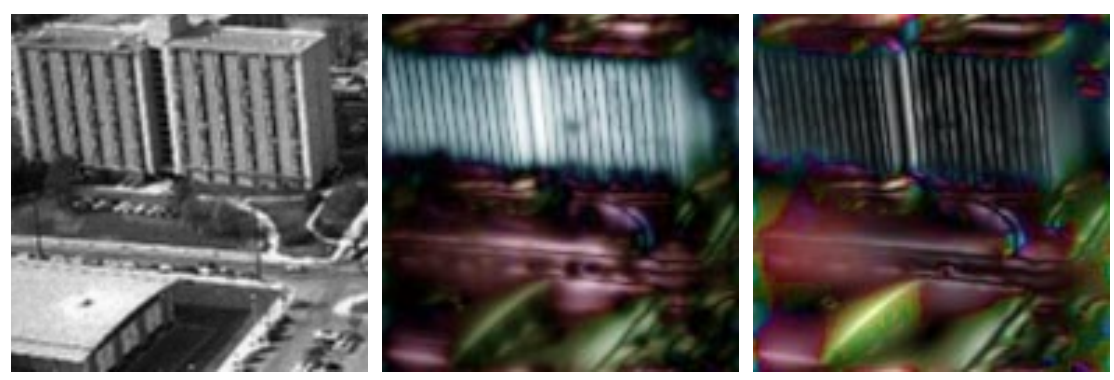

Fig. 8. An image (left) and the orientation distribution visualizations with using variance (middle) and scale summarized gradient (right). Color hue maps angular direction.

and dominant spatial features reside. Images that do not have a similar visual content at coarser scales may be disregarded, while the search could be continued for the rest of the images at a finer scale. This would result in a reduced computational cost for generic queries, under the assumption that large scales dominate image impression. Inversely, and depending on the field of application (e.g. texture similarity), detail may be primarily investigated by focusing at fine scale structure.

\section{Conclusions - Future Work}

In this work, scale was studied as a component of image feature detection and it was argued that task performance is enhanced if carried out in more than one scales. A framework was introduced for summarizing feature contributions from all scales, into a single scaleless representation, and a methodology was presented for the robust classification of features by scale of appearance.

The application of SSR and SF in the context of image retrieval by content was studied through the computation of feature distributions at multiple scales. The detection of dominating feature distributions gives a more qualitative description of an image's primitive visual content. Through scale classification of image features, queries may target more abstract scene content or image detail.

It is speculated that SSR and SF are compatible with neurophysiological results. Evidence of parallel multiscale analysis of structure is observed in primate vision, through spatial frequency selectivity in hypercolumns located in the striate cortex [7]. At a higher level, different spatial frequency responses are to be linearly (due to the computational nature of neurons) combined towards a single image perception. Focusing on certain scales for feature extraction is analogous to the attentional activation of winner-take-all networks [2].

Comparing the computational requirements of SSR and SF with scale space feature detection, it should be noted that the SSR approach does not require feature tracking, as employed in typical scale space methodologies $[1,6]$. The memory used by SSR techniques is simply proportional to the precision needed 
by the application. Computing multiscale feature information can be simultaneously parrallelized in two ways: (i) processing each scale's information independently (ii) processing separately local pixel information. Nevertheless, a serial methodology execution, of feature detection, in 256 x 256 image at eight different scales takes about half a minute, on an average personal computer.

Future work includes the study of color features, work on feature distribution clustering, the study of appropriate similarity measures, and the adoption of perceptual grouping rules for the formation of visual entities.

\section{References}

1. F. Bergholm. Edge focusing. IEEE Transactions on Pattern Analysis and Machine Intelligence, 9:726-741, 1987.

2. C. Koch D.K. Lee, L. Itti and J. Braun. Attention activates winner-take-all competition among visual filters. Nature Neuroscience, 2(4):375-381, 1999.

3. J. Puzicha et al. Empirical evaluation of dissimilarity measures for color and texture. In Proceedings of the International Conference on Computer Vision, 1999.

4. K. Kanatani. Detection of surface orientation and motion from texture by stereological technique. Journal of Artificial Intelligence, 23:213-237, 1984.

5. Jan J. Koenderink and Andrea J. van Doorn. The structure of locally orderless images. International Journal of Computer Vision, 31(2/3):159-168, 1999.

6. T. Lindeberg. Scale-Space Theory in Computer Vision. The Kluwer International Series in Engineering and Computer Science. Kluwer Academic Publishers, Boston, USA, 1994.

7. De Valois R. and De Valois K. Spatial Vision. Oxford Science Publications, Oxford, 1988.

8. Karl Rohr. Modelling and identification of characteristic intensity variations. Image and Vision Computing, 10(2):66-76, 1992.

9. S. Santini and R. Jain. Similarity matching. IEEE Transactions on Pattern Analysis and Machine Intelligence, 21(9):871-883, 1999.

10. S. Sarkar and K.L. Boyer. Perceptual organization in computer vision: A review and a proposal for a classificatory structure. SMC, 23:382-399, 1993.

11. J. R. Smith and S.-F. Chang. Integrated spatial and feature image query. Multimedia System Journal. Springer-Verlag., 7(2):129-140, 1999.

12. J. Weickert, S. Ishikawa, and A. Imiya. On the history of Gaussian scale-space axiomatics. In Jon Sporring, Mads Nielsen, Luc Florack, and Peter Johansen, editors, Gaussian Scale-Space Theory, chapter 4, pages 45-59. Kluwer Academic Publishers, Dordrecht, The Netherlands, 1997. 